\title{
Pointwise taut Riemannian manifolds
}

\author{
Michael Leitschkis
}

\begin{abstract}
In the present work, we investigate pointwise taut Riemannian manifolds by using methods from Riemannian geometry, Morse theory and topology.

Using ideas of Terng and Thorbergsson, we extend a result of Warner and show that a compact simply connected pointwise taut $n$-dimensional manifold is homeomorphic to a compact rank one symmetric space, if the first conjugate locus of a point is of constant multiplicity. We apply this result to study compact simply connected pointwise taut manifolds in dimensions three and four.
\end{abstract}

Mathematics Subject Classification (2000). 53C25.

Keywords. Pointwise taut, Blaschke, cut locus.

\section{Introduction}

Let $N$ be a complete Riemannian manifold. A point $q \in N$ is called taut, if the energy functional $E_{p}: P(N, q \times p) \rightarrow \mathbb{R}$ is perfect for every $p \in N$ that is not conjugate to $q$ along some geodesic. We call a complete manifold pointwise taut, if each of its points is taut.

The purpose of the present work is to study pointwise taut manifolds, especially in low dimensions, by using methods from Riemannian geometry, Morse theory and topology. Some of the frequently used notions are those of conjugate and cut loci, critical manifolds and path spaces.

According to a theorem of Bott and Samelson ([BS]), compact simply connected symmetric spaces are pointwise taut. All known examples of compact simply connected pointwise taut manifolds being symmetric, one is led to the natural question of whether the converse of their result holds. An affirmative answer to this question would imply the Blaschke conjecture (see Theorem 1.6 due to Terng and Thorbergsson).

For compact simply connected pointwise taut manifolds with the additional property that their first conjugate points be of the same multiplicity, we obtain the following 
Theorem. Let $M$ be a compact simply connected pointwise taut $n$-dimensional Riemannian manifold. If there exists a point $p$ in $M$ for which each point of the first conjugate locus $C_{1}(p)$ in $T_{p} M$ has the constant multiplicity $k$, then one of the following holds:

(i) $k=n-1$, and $M$ is isometric to the standard sphere $S^{n}$ with constant sectional curvature.

(ii) $k=1, n=2 \lambda$ for $\lambda=2,3,4, \ldots$ and $M$ is homeomorphic with the complex projective space $P^{\lambda}(\mathbb{C})$. If $n>4$, then $M$ is even diffeomorphic with $P^{\lambda}(\mathbb{C})$.

(iii) $k=3, n=4 \lambda$ for $\lambda=2,3,4, \ldots$ and $M$ is homeomorphic with the quaternionic projective space $P^{\lambda}(\mathbb{H})$.

(iv) $k=7, n=16$ and $M$ is homeomorphic with the projective Cayley plane $P^{2}(\mathbb{O})$.

This result constitutes the foundation of our subsequent studies. By applying it to the three-dimensional case, we prove the following

Theorem. Let $M$ be a compact simply connected pointwise taut three-manifold. Then $M$ is isometric to the standard sphere $S^{3}$.

As one would expect, the four-dimensional case is much more difficult. From dimension four on, the first conjugate locus of a point in a pointwise taut manifold can include points of different multiplicities. For example, multiplicities one and two appear in the first conjugate locus of a point in $S^{2} \times S^{2}$. Therefore, a direct application of our results from the constant multiplicity case is not possible. Though, techniques from that case can be used locally, when we consider open subsets of constant multiplicity in the first tangent conjugate locus of a point. We assume our pointwise taut manifolds to be compact and simply connected, because then the cut locus of a point coincides with the first conjugate locus, as was shown by Terng and Thorbergsson ([TT]). Their result leads us to some facts about the cut locus, which in turn allow us to draw conclusions about the geometry of pointwise taut four-manifolds, i.a. the following

Theorem. Let $M$ be a compact simply connected pointwise taut four-manifold. Then each point of $M$ is conjugate to itself along a suitable geodesic.

In Section 1, we give some preliminaries on taut immersions into complete Riemannian manifolds, stressing the case of taut points, and quote the basic results on pointwise taut manifolds that are due to Terng and Thorbergsson ([TT]).

In Section 2, the constant multiplicity case is studied, using Warner's work ([Wa1], [Wa2]) and various results about Blaschke manifolds. In Sections 3 and 4, we apply our results on the constant multiplicity case to compact simply connected pointwise taut manifolds of dimensions three and four respectively. 
The results presented in this article have been part of my doctoral thesis. I would like to thank my teacher, Professor G. Thorbergsson, who suggested this topic to me, for his long-term guidance, encouragement and support of my work. Thanks are also due to Professor W. Ziller, who supervised my work at the University of Pennsylvania in Philadelphia for a year, and to the DAAD, whose grant made my visit to Philadelphia possible.

\section{Preliminaries}

We begin by recalling that a Morse function on a manifold $M$ is called perfect with respect to a field $F$, if the number of index $k$ critical points is equal to the $k$-th Betti number of $M$ with respect to $F$ for all $k$.

A submanifold $M$ of $\mathbb{R}^{n}$ is called taut if there is a field $F$ such that, for generic $a \in \mathbb{R}^{n}$, the squared distance function $f_{a}: M \rightarrow \mathbb{R}$ is perfect with respect to $F$. This terminology was introduced by Carter and West ([CW]), but some results on taut submanifolds actually go back to Bott ([Bo2]) and Bott and Samelson ([BS]).

A definition similar to the one above can be also used for submanifolds of $S^{n}$. In fact, one can call a submanifold $M$ of $S^{n}$ taut if a squared distance function $f_{a}$ of $M$ is a perfect Morse function for generic $a$. Though, this notion of tautness cannot be directly generalized for submanifolds in arbitrary Riemannian manifolds $N$. One of the problems is that the squared distance function $f_{a}: N \rightarrow \mathbb{R}$ defined by $f_{a}(x)=d(p, x)^{2}$ is not differentiable if the cut locus of $a$ is not empty. In $N=S^{n}$, this is not a problem since a submanifold of $S^{n}$ does not meet the cut locus of $a$ for generic $a$. But this is not true for a general manifold $N$.

Using a different approach, Grove and Halperin ([GH]) and, independently of them, Terng and Thorbergsson ([TT]) defined a general notion of a taut immersion into a complete Riemannian manifold. It was proved ([TT]) that for submanifolds of the Euclidean space and those of the sphere, the new tautness definition coincides with the one previously known. Now we are going to introduce this generalized tautness notion, using the exposition in the paper [TT].

Let $(N, g)$ be a Riemannian manifold and $\phi: M \rightarrow N$ an immersion. For $B \subset$ $N \times N$, denote by $P(N, B)$ the set of all $H^{1}$-paths $\gamma$ in $N$ such that $(\gamma(0), \gamma(1)) \in B$. Notice that a path is $H^{1}$ if it is absolutely continuous and the norm of the derivative is square integrable. For a fixed $p \in N$, let $\pi: P(N, N \times p) \rightarrow N$ be the fibration defined by $\pi(\gamma)=\gamma(0)$, and let $P(N, \phi \times p)$ denote $\phi^{\star}(P(N, N \times p))$, i.e., the space of pairs $(q, \gamma)$ such that $q \in M$ and $\gamma$ is a $H^{1}$-path $\gamma:[0,1] \rightarrow N$ such that $(\gamma(0), \gamma(1))=(\phi(q), p)$. The space $P(N, \phi \times p)$ is a Hilbert manifold ([Pa]). If $M \in N$ and $\phi$ is the inclusion map, then $P(N, \phi \times p)$ is isomorphic to the space 
$P(N, M \times p)$. Let

$$
E_{p}: P(N, \phi \times p) \rightarrow \mathbb{R}, \quad E_{p}(q, \gamma)=\int_{0}^{1}\left\|\gamma^{\prime}(t)\right\|^{2} d t
$$

be the energy functional. Then it is well known that $(q, \gamma) \in P(N, \phi \times p)$ if and only if $\gamma$ is a geodesic normal to $\phi(U)$ at $\phi(q)=\gamma(0)$ parametrized proportionally to arc length, where $U$ is a neighborhood of $q$ on which $\phi$ is injective. It is also well known that $E_{p}$ is a Morse function if and only if $p$ is not a focal point of $M$. Notice that we do not require that the levels of critical points of a Morse function are different, only that all critical points are non-degenerate. The Morse index theorem says that the index of $E_{p}$ at a critical point $(q, \gamma)$ is the sum of integers $m$ such that $\gamma(t)$ is a multiplicity $m$ focal point of $M$ with respect to $q$ with $0<t<1$.

Let $\mu_{k}$ denote the number of index $k$ critical points of $E_{p}$ in $P(N, \phi \times p)$, and let $b_{k}$ denote the $k$-th Betti number of $P(N, \phi \times p)$. It is known that $E_{p}$ is bounded below and satisfies the Palais-Smale condition. So if $p$ is not a focal point of $M$, then for an arbitrary number $r$ the number $\mu_{k}(r)$ of critical points of index $k$ on the subset

$$
P(N, \phi \times p)^{r}=\left\{(q, \gamma) \in P(N, \phi \times p) \mid E_{p}(q, \gamma) \leq r\right\}
$$

is finite for all $k$ (see [PS]). The well-known Morse inequalities $\mu_{k}(r) \geq b_{k}(P(N$, $\phi \times p)^{r}$ ) hold for all $k$ and $r$. The functional $E_{p}$ is called perfect if each of these inequalities is an equality, that is if $\mu_{k}(r)=b_{k}\left(P(N, \phi \times p)^{r}\right)$ for all $k$ and $r$.

Remark. In the sequel, we will deal with a special case, in which $M$ is just a point $q \in N$. In this situation, we will not use the rather complicated notation $P(N, q \times p)$ and denote this path space by $\Omega_{p q}(N)$ instead. In the literature, this notation is often used to denote the space of continuous paths from $p$ to $q$ in $N$. We can use the same notation for our space of $H^{1}$-paths, since these spaces are homotopy equivalent ([Pa]). Moreover, we set

$$
\Omega_{p q}^{r}(N)=\left\{\gamma \in \Omega_{p q}(N) \mid E_{p}(\gamma) \leq r\right\}
$$

and

$$
\Omega_{p q}^{r-}(N)=\left\{\gamma \in \Omega_{p q}(N) \mid E_{p}(\gamma)<r\right\}
$$

Definition 1.1. Let $N$ be a complete Riemannian manifold. An immersion $\phi: M \rightarrow N$ of $N$ is called taut if there is a field $F$, so that the energy functional $E_{p}: P(N, \phi \times p) \rightarrow \mathbb{R}$ is perfect with respect to $F$ for every $p \in N$ that is not a focal point of $M$. In particular, a point $q \in N$ is called a taut point if $\{q\}$ is a taut submanifold of $N$, i.e., $E_{p}: \Omega_{q p}(N) \rightarrow \mathbb{R}$ is perfect for every $p \in N$ that is not conjugate to $q$ along some geodesic. 
Now we are going to turn our attention to the energy functional $E_{p}$, where $p$ is a focal point of $M$. According to the above, this $E_{p}$ is not a Morse function. Therefore, the critical points of $E_{p}$ need not be isolated. Among such functions, one distinguishes the so-called Morse-Bott functions, which are defined by the property that each connected component of the set of critical points is a non-degenerate critical submanifold (of $\Omega_{q p}(N)$ in our situation). The non-degeneracy of a critical submanifold means that all critical points in it have the same nullity, which equals the dimension of the submanifold. For further information on critical submanifolds, one can consult [Bo1]. The concept of critical submanifolds was applied by Ozawa ([Oz]) to study taut submanifolds in Euclidean spaces and spheres. Terng and Thorbergsson showed in [TT] that Ozawa's result carries over to taut immersions into complete Riemannian manifolds. They proved, that for a taut immersion into a complete Riemannian manifold, the energy functional $E_{p}$ is a Morse-Bott function for an arbitrary point $p$. We formulate their result for the special case of taut points, which is to be studied in the present work.

Theorem 1.2. Let $N$ be a complete Riemannian manifold with a taut point $q$ and $p$ a point in $N$ conjugate to $q$ along a geodesic $\gamma$. Then the critical point $\gamma$ of $E_{p}: \Omega_{q p} \rightarrow \mathbb{R}$ lies in a non-degenerate connected critical submanifold $\mathrm{Cr} \subset \Omega_{q p}$ of dimension $m$, where $m$ is the multiplicity of $p$ (or, in other words, the nullity of $\gamma$ as a critical point). Moreover, the geodesics from $q$ to $p$, which belong to $\mathrm{Cr}$, have the same length.

Now we give some basic information about the pointwise taut manifolds.

Definition 1.3. We call a Riemannian manifold $(M, g)$ pointwise taut, if each of its points is taut.

In terms of this definition, a result due to Bott and Samelson ([BS]) signifies that any symmetric space is pointwise taut. The field with respect to which the symmetric spaces are pointwise taut is $\mathbb{Z}_{2}$. Of course, some of these spaces are also pointwise taut with respect to other fields.

Up to now, all known examples of compact pointwise taut manifolds are symmetric. Therefore, one is tempted to conjecture that there are no other compact pointwise taut manifolds. Although it does not seem possible to prove such a general theorem, some encouraging partial results have been achieved by Terng and Thorbergsson and motivated us to continue research in this direction. Now we are going to quote their results from [TT], which establish a connection between pointwise taut manifolds and Blaschke manifolds. We remind the reader that the latter can be defined in the following manner:

Let $p$ and $q$ be distinct points in a complete Riemannian manifold $M$. Set $d=$ $d(p, q)$. Then the link from $p$ to $q$ is defined to be the set $\Lambda(p, q)$ of unit vectors 
$X=\gamma^{\prime}(d)$ in $T_{q}(M)$ where $\gamma:[0, d] \rightarrow M$ is a length minimizing geodesic between $p$ and $q$. A compact Riemannian manifold is said to be a Blaschke manifold at $p$ for every point $q$ in the cut locus $\operatorname{Cut}(p)$ of $p$ the link $\Lambda(p, q)$ is a totally geodesic sphere in the unit sphere of $T_{q} M$. A Riemannian manifold is said to be Blaschke, if it is Blaschke at all of its points.

Theorem 1.4. Let $\left(M^{n}, g\right)$ be a simply connected compact Riemannian manifold with a taut point $p$.

(i) If the first two non-vanishing Betti numbers of the based loop space are $b_{0}$ and $b_{n-1}$ and $p \in M$, then $(M, g)$ is Blaschke at $p$ and $M$ is homeomorphic to $S^{n}$.

(ii) If a point $q$ is the first conjugate point to $p$ along a geodesic, so that the multiplicity of $q$ is $n-1$, then $(M, g)$ is Blaschke at $p$ and $M$ is homeomorphic to $S^{n}$.

Remark. In [TT], the first part of this theorem is stated explicitly, whereas the second statement follows trivially from the proof of the first one. We state the theorem in this way because it is mainly the second claim which we will use in our work.

Theorem 1.5. Let $\left(M^{n}, g\right)$ be a simply connected compact Riemannian manifold. Suppose the first three nonvanishing Betti numbers $b_{i}$ of the based loop space of $M$ are $b_{0}, b_{a}$ and $b_{a+n-1}$ for some $1 \leq a \leq n-1$ and $b_{a}=1$. If $p$ is taut in $M$, then $(M, g)$ is Blaschke at the point $p$. In particular, if all points are taut, then $(M, g)$ is Blaschke.

Remark. The homology groups under consideration above are those with coefficients in $F$, where $F$ is the field with respect to which the tautness assumption holds.

Terng and Thorbergsson also proved the following

Theorem 1.6. Let $(M, g)$ be a simply connected Blaschke manifold. Then it is pointwise taut.

It can be easily checked that all compact symmetric spaces of rank one are Blaschke. No other examples of Blaschke manifolds are known. In fact, the socalled Blaschke conjecture (never conjectured by Blaschke in this generality) states that every Blaschke manifold is isometric to a compact symmetric space of rank one.

The basic facts about Blaschke manifolds, which we have gathered, enable us to better understand the theorems of Terng and Thorbergsson quoted above. Because Blaschke manifolds are conjectured to be compact rank one symmetric spaces, Terng and Thorbergsson had to assume in Theorem 1.4 and Theorem 1.5 that the based loop spaces of the manifolds considered there have the right Betti numbers in order to have 
any reasonable chance of proving those manifolds to be Blaschke. Their successful investigation of this situation means that, when studying a manifold whose based loop space has the same Betti numbers as that of a compact rank one symmetric space, we can use vast information gathered about Blaschke manifolds. We will quote some advanced information about them in the later sections whenever necessary.

If we knew that pointwise taut manifolds are symmetric spaces, then Theorem 1.6 would imply the Blaschke Conjecture, which is still unsettled. Thus, we will not try to prove that a pointwise taut manifold is isometric to a symmetric space. Even the objective of showing that a compact pointwise taut manifold is homeomorphic to a symmetric space is not achievable in general, but we will solve this problem under an additional assumption on the conjugate locus in Section 2.

We conclude this section with an elementary topological remark clarifying the role of the based loop space in the theorems above. Indeed, it was the path space $\Omega_{p q}$ that was mentioned in the definition of tautness in Section 2, not the based loop space. But, due to a well-known result by Serre ([Se], p. 480), the path spaces $\Omega_{x y}$ and $\Omega_{z w}$ are of the same homotopy type, for arbitrary points $x, y, z, w$.

\section{The constant multiplicity case}

Let $M^{n}$ be a compact simply connected pointwise taut manifold. In this section, we are going to assume that there exists a point $p \in M$ for which each point of the first conjugate locus $C_{1}(p) \subset T_{p} M$ has the constant multiplicity $k$.

The case $2 \leq k \leq n-1$ has been treated by Warner ([Wa2]). In fact, without making any tautness assumption, he proved the following result.

Theorem 2.1. Let $M$ be a compact simply connected $n$-dimensional Riemannian manifold, and let $k$ be an integer with $2 \leq k \leq n-1$. If there exists a point $p$ in $M$ for which each point of the first conjugate locus $C_{1}(p)$ in $T_{p} M$ has the constant multiplicity $k$, then one of the following holds:

(i) $k=n-1$, and $M$ is homeomorphic with the sphere $S^{n}$.

(ii) $k=3$, and $n=4 \lambda$ for $\lambda=2,3,4, \ldots$ and the integral cohomology ring of $M$ is isomorphic to that of the quaternionic projective space $P^{\lambda}(\mathbb{H})$.

(iii) $k=7$, and $n=16$, and the integral cohomology ring of $M$ is isomorphic to that of the projective Cayley plane $P^{2}(\mathbb{O})$.

Because of this theorem, the main object of our studies is the case $k=1$. In this case, we want to prove that $M$ is even-dimensional and that the integral homology of $M$ is isomorphic to that of the complex projective space of the right dimension. After achieving this goal, we will use our tautness assumption again to sharpen the results in each case. 
We are going to take advantage of the general strategy which was used by Warner to prove the theorem cited above. Warner had to assume $k \geq 2$, because some arguments of his proof break down in the case $k=1$, but we will overcome these problems by using the pointwise tautness of $M$. We begin by introducing some terminology and results of Warner ([Wa1]), which will be used in the sequel. First we cite an important theorem about the exponential map of a manifold.

Theorem 2.2. Let $M$ be a Riemannian manifold, $p \in M$ be arbitrary. Consider the exponential map $\exp _{p}: T_{p} M \rightarrow M$. Then for each non-zero point $x$ in $T_{p} M$ there exists a convex neighborhood $U$ of $x$ such that the number of conjugate points (counted with multiplicities) on $r \cap U$, for each ray through the origin which intersects $U$, is constant and equals the multiplicity of $x$ as a conjugate point to $p$ along $\exp _{p}(t x)$.

Remark. This theorem, which will help us understand the structure of the conjugate locus of our pointwise taut manifolds, is not explicitly stated in [Wa1] in the above form. In [Wa1], Warner introduces a notion of regular exponential maps, which in particular satisfy Theorem 2.2 by their definition. He proceeds in that article by showing that the usual Riemannian exponential map is regular.

Definition 2.3. A conjugate point $x \in T_{p} M$ is called regular if there exists a neighbourhood $U$ of $x$ such that each ray through the origin of $T_{p} M$ contains at most one point in $U$ which is a conjugate point. A conjugate point which is not regular is called a singular point.

We let $C^{R}(p)$ denote the regular points in $C_{1}(p)$ and $C^{S}(p)$ denote the singular points. Theorem 2.2 implies the following useful

Lemma 2.4. The regular conjugate locus $C^{R}(p)$ is open. Therefore, the singular conjugate locus is closed. Moreover, all conjugate points of multiplicity one are regular.

Examples 2.5. We consider two four-manifolds, which we will encounter in Section 4 . We think of these manifolds as endowed with their standard metrics.

(i) $M=P^{2}(\mathbb{C})$. It is well known that each first conjugate point of the complex projective space has multiplicity one. Lemma 2.4 implies that the first conjugate locus consists of regular points only.

(ii) $M=S^{2} \times S^{2}$. Let $(p, q)$ be an arbitrary point of $M$ and $(V, W) \in T_{(p, q)} M$ be an arbitrary tangent vector. The multiplicity of the first conjugate point with respect to $(p, q)$ in direction $(V, W)$ can be easily computed. One obtains the following results: is $\|V\|>\|W\|$, then the first conjugate point to $(p, q)$ in the direction $(V, W)$ is $(-p, r)$ with $r \neq-q$. The multiplicity of this conjugate 
point is one. Analogously, if $\|W\|<\|V\|$, then the first conjugate point to $(p, q)$ in the direction $(V, W)$ is $(r,-q)$ with $r \neq-p$. Again, the multiplicity of this conjugate point is one. Eventually, if $\|V\|=\|W\|$, then the first conjugate point to $(p, q)$ in the direction $(V, W)$ is $(-p,-q)$. The multiplicity of this conjugate point equals two.

The regular conjugate locus admits a particularly nice description which was given by Warner in [Wa1].

Theorem 2.6. Let $M^{n}$ be a Riemannian manifold, $p \in M$ be arbitrary. Then the regular conjugate locus $C^{R}(p)$ is an open everywhere dense subset of $C(p)$ which can be given a manifold structure of dimension $n-1$ such that the inclusion $i: C^{R}(p) \rightarrow T_{p} M$ is a submanifold with the relative topology, and such that $\left(T_{p} M\right)_{x}=\left(C^{R}(p)\right)_{x} \oplus r_{x}$ for every $x \in C^{R}(p)$.

Let $x \in C^{R}(p)$, let $\exp _{p}: T_{p} M \rightarrow M$ be the exponential map. We define $T(x)$ to be the subspace of the null space $N(x)$ of $d \exp _{p}(x)$ tangential to the regular conjugate locus at $x$, i.e., $T(x)=N(x) \cap\left(C^{R}(p)\right)_{x}$.

Consider an open connected submanifold $C$ of the regular conjugate locus $C^{R}(p)$. The multiplicity of the conjugate points comprising $C$ is a constant $k$. The null space $N(x)$ of $d \exp _{p}(x)$ intersects the tangent space $C_{x}$ to $C$ in either a $k$ or $(k-1)$ dimensional subspace which we have denoted by $T(x)$. Let $C^{k}$ be the subset of points $x$ in $C$ where the dimension of $T(x)$ is $k$ and $C^{k-1}$ the subset where the dimension of $T(x)$ is $k-1$. For the case $k \geq 2$, Warner ([Wa1]) proved the following

Theorem 2.7. The set $C^{k-1}$ is empty for $k \geq 2$. That is, if $x$ is a regular conjugate point of the exponential map $\exp _{p}: T_{p} M \rightarrow M$ and if the multiplicity of $x$ is $\geq 2$, then the null space $N(x)$ of $d \exp _{p}(x)$ is contained in the tangent space $\left(C^{R}(p)\right)_{x}$ to the regular conjugate locus at $x$.

We will need an analogous theorem for $k=1$, but in this case Warner's proof breaks down. We are going to prove the desired result in a different way, using our assumption that $M$ is pointwise taut. Moreover, we can restrict ourselves to the first conjugate locus, because we will not need a more general result.

Proposition 2.8. Let $M$ be a compact simply connected pointwise taut manifold and $p \in M$ arbitrary. Then the set $C^{0}(p) \cap C_{1}(p)$ is empty. That is, if $x \in T_{p} M$ is a regular first conjugate point and if the multiplicity of $x$ is one, then the null space $N(x)$ of $d \exp _{p}(x)$ is contained in the tangent space $\left(C^{R}(p)\right)_{x}$ to the regular conjugate locus at $x$. 
Proof. Let $x \in T_{p} M$ be a regular first conjugate point of multiplicity one. Let $c(t)$ be the line segment between 0 and $x$ in $T_{p}(M)$ and $q=\exp _{p}(x)$. According to Theorem 1.2, the geodesic segment $\exp _{p}(c(t))$, viewed as a point of the path space $\Omega_{p q}(M)$, is contained in a one-dimensional compact critical manifold $\mathrm{Cr}$. All the points of this critical manifold are geodesic segments of the same length. Moreover, $\mathrm{Cr}$ is homeomorphic to $S^{1}$ according to the well-known classification of 1-manifolds. We can think of $C r$ as of a closed curve $\sigma$ in $C_{1}(p)$ with $\sigma(0)=x=\sigma(1)$, with the correspondence between $C r$ and $\sigma$ provided by the exponential map $\exp _{p}$. All we need to prove now is the differentiability of $\sigma$.

To show the latter, we use the proof of Theorem 1.2, which is due to Terng and Thorbergsson ([TT]). They work with the standard finite dimensional approximation of the path space $\Omega_{p q}(M)$ on the product of a suitable number of copies of $M$. Since we assumed that $q$ is contained in the first conjugate locus, one copy of $M$ is actually enough in our case. The geodesic segments, which make up $C r \subset \Omega_{p q}(M)$, are approximated in $M$ by their midpoints. We denote the "approximating manifold" by $\overline{C r}$, which is a closed curve in $M$. The proof of Terng and Thorbergsson establishes the differentiability of this curve. Therefore, the closed curve $\bar{\sigma}=\exp _{p}^{-1}(\overline{C r})$ in $T_{p} M$ is differentiable as well. This implies the differentiability of the curve $\sigma=2 \bar{\sigma}$.

Another important result which was shown by Warner for the case $k \geq 2$ can be stated as follows:

Theorem 2.9. Let $M$ be a simply connected complete Riemannian manifold, and let $p \in M$. Suppose that, whenever there exists a first conjugate point to $p$ along a geodesic issuing from $p$, that conjugate point has multiplicity $\geq 2$. Then the cut locus and the first conjugate locus in $T_{p} M$ coincide.

This statement was proven by Warner without any tautness assumption, but his proof is not valid in the $k=1$ case. Using tautness, Terng and Thorbergsson ([TT]) obtained a similar result without assuming $k \geq 2$ :

Theorem 2.10. Suppose $M$ is a complete simply connected Riemannian manifold with a taut point $p$. Then the first conjugate point of $p$ along a geodesic coincides with the cut point of $p$ along that geodesic and vice versa.

Now we are ready to state and prove the key result of this section. The proof resembles Warner's proof of Theorem 2.1 ([Wa2]) closely. Nevertheless, we do not omit it, because some statements which are shown on course of this proof (for example, Lemma 2.14) are also interesting in their own right and used in Section 4.

Theorem 2.11. Let $M$ be a compact simply connected $n$-dimensional manifold. If there exists a taut point $p$ in $M$ for which each point of the first conjugate locus in 
$T_{p} M$ has the constant multiplicity one, then $n$ is even ( say, $n=2 \lambda$ with a $\lambda \in \mathbb{Z}$ ) and the integral cohomology ring of $M$ is isomorphic to that of $P^{\lambda}(\mathbb{C})$.

Proof. As usual, we let $C_{1}(p)$ denote the first conjugate locus in $T_{p} M$. It is well known (for example, see $[\mathrm{dC}]$, p. 272) that there is a homeomorphism $f$ between the unit sphere $S \in T_{p} M$ and the tangent cut locus, which coincides with $C_{1}(p)$, as we know from Theorem 2.10. According to Lemma 2.4, $C_{1}(p)$ is a connected component of the regular conjugate locus. By Theorem 2.6, $C_{1}(p)$ has a unique manifold structure for which $\left(C_{1}(p), i\right)$ is a submanifold of $T_{p} M$, where $i$ is the inclusion map. With this manifold structure on $C_{1}(p), f$ becomes a diffeomorphism.

Let $x \in C_{1}(p)$ be arbitrary. We let $C_{1}(p)_{x}$ denote the tangent space to the first conjugate locus at $x$. Moreover, let $S_{x}$ be the subspace of $C_{1}(p)_{x}$ tangent to the sphere about the origin in $T_{p} M$, passing through $x$ and $N_{x}$ be the kernel of $d \exp _{p} \mid\left(T_{p} M\right)_{x}$. According to Proposition 2.8, $N_{x}$ is contained in $C_{1}(p)_{x}$. Thus the $N_{x}$ define a onedimensional distribution $\mathcal{N}$ on $C_{1}(p)$. Moreover, $N_{x}$ is contained in $S_{x}$ because of the Gauss lemma. We obtain

$$
N_{x} \subset S_{x} \subset C_{1}(p)_{x}
$$

with dimensions $1, n-1$ or $n-2$, and $n-1$ respectively. The following lemma can be obtained by arguing exactly like Warner did in the proof of Theorem 2.1. But in the case $k=1$, we can also use the classification of one-dimensional manifolds.

Lemma 2.12. Each maximal connected integral manifold of the distribution $\mathcal{N}$ is diffeomorphic with $S^{1}$.

Following his proof further, we can easily show the following

Lemma 2.13. Let $x \in \exp _{p}\left(C_{1}(p)\right)$ be arbitrary. Then $\exp _{p}^{-1}(x) \cap C_{1}(p)$ consists of a single maximal connected integral manifold of $\mathcal{N}$.

Since the exponential map is $1: 1$ on the integral manifolds of $N$, it follows that the cut locus $\operatorname{Cut}(p)$ in the relative topology has a unique differentiable structure such that $(\operatorname{Cut}(p), i)$ is a submanifold of $M$ where $i$ is the inclusion map. Moreover, the map

$$
\exp _{p} \mid C_{1}(p): C_{1}(p) \rightarrow \operatorname{Cut}(p)
$$

is $C^{\infty}$. According to Wolf ([Wo]), this map is a differentiably locally trivial fiber bundle, its structural group being the group of all diffeomorphisms of the fiber in the compact open topology. The total space $C_{1}(p)$ is diffeomorphic with $S^{n-1}$ and the fiber is $S^{1}$. The base of this fibering is $\operatorname{Cut}(p)$. Obviously, the dimension of $\operatorname{Cut}(p)$ is $n-2>0$. From [Wa2], we know that $\operatorname{Cut}(p)$ is simply connected, because $M$ is. It follows from the Gysin sequence that the dimension of $\operatorname{Cut}(p)$ must be even, 
say $2(\lambda-1)$ (whence $n=2 \lambda)$ and that the integral cohomology ring $H^{\star}(\operatorname{Cut}(p) ; \mathbb{Z})$ is a truncated polynomial ring with one generator $x$ in dimension 2 and the relation $x^{\lambda}=0$.

Thus, according to Warner ([Wa2]), the integral cohomology group $H^{\star}(M ; \mathbb{Z})$ is a free abelian group with free basis $\left\{1, y, \ldots, y^{\lambda-1}, z\right\}$, where $y \in H^{2}(M ; \mathbb{Z})$ and $z$ is a generator of $H^{n}(M ; \mathbb{Z})$. To finish the proof of Theorem 2.11 , we have to verify that the ring structure is correct. In other words, we have to show that $z= \pm y^{\lambda}$. We fix an orientation on $M$ and denote by $D$ the Poincaré duality. Since $H_{\star}(M ; \mathbb{Z})$ has no torsion, we have an isomorphism

$$
\phi: H_{i}(M ; \mathbb{Z}) \rightarrow \operatorname{Hom}\left(H_{n-i}(M ; \mathbb{Z})\right),
$$

where $\phi(\sigma)(\tau)=D(D \sigma \cup D \tau)$ for $\sigma \in H_{i}(M ; \mathbb{Z})$ and $\tau \in H_{n-i}(M ; \mathbb{Z})$. So there exists $\sigma \in H_{n-2}(M ; \mathbb{Z})$ such that $\phi_{\sigma}(D y)=1$; i.e., $D \sigma \cup y= \pm z$. Since there is an $m \in \mathbb{Z}$ so that $D \sigma=m y^{\lambda-1}$, we obtain $m y^{\lambda-1}= \pm z$. But there is an $l \in \mathbb{Z}$ so that $y^{\lambda}=l z$. Therefore $m n= \pm 1$, whence $m= \pm 1$ and $z= \pm y^{\lambda}$.

Before proceeding further, we draw a consequence from the proof above, which will later help us study those pointwise taut manifolds to which our current "constant multiplicity" assumption does not apply. To make a statement about that general situation, we use the definition of regular conjugate points to see that on any connected component of the regular conjugate locus, the multiplicity of the conjugate points is constant. Thus we obtain the following

Lemma 2.14. Let $M^{n}$ be a compact simply connected manifold with a taut point $p$. Let $A$ be a connected component of the regular conjugate locus $C^{R}(p)$ in the first tangent conjugate locus $C_{1}(p)$ and set $\mathrm{Cut}_{A}=\exp _{p}(A)$. Then $\mathrm{Cut}_{A}$ is an $(n-k-1)$ dimensional submanifold of $M$ and $\exp _{p}: A \rightarrow \mathrm{Cut}_{A}$ is a differentiable locally trivial fibration with fiber $S^{k}$, where $k$ is the common multiplicity of the conjugate points in $A$.

Our next goal is to strengthen the result of Theorem 2.11 and those of Theorem 2.1 by using our pointwise tautness assumption once again. We begin with the case $k=1$. According to Theorem 2.11, the integral cohomology of $M$ is isomorphic to that of the complex projective space of the right dimension. Poincaré Duality implies that the same isomorphy holds in homology. Let $F$ be the field with respect to which $M$ is pointwise taut. Due to the Universal Coefficients Theorem, the homologies with coefficients in a field $F$ are isomorphic as well. Now we want to show that $M$ is in fact homeomorphic to that space. We begin by quoting a well-known topological result which will give us the information we need on the Betti numbers of $\Omega M$ :

Proposition 2.15. Let $M^{n}$ be a differentiable manifold, so that the homology of $M$ with coefficients in a field $F$ is isomorphic to that of a compact rank one symmetric 
space. Then the homology of the based loop space $\Omega(M)$ with coefficients in $F$ is isomorphic to that of the based loop space of the symmetric space.

Remark. One of the ways of proving this proposition is by applying the Leray-Serre spectral sequence to the path-loop fibration. For the spherical case, this calculation can be found in $[\mathrm{BT}]$, p. 204. The remaining cases can be handled in the same way.

The proposition above and the well-known data on the Betti numbers of compact rank one symmetric spaces together imply the following obvious conclusion, which we will use later. If $M$ has the homology of $P^{\lambda}(\mathbb{C}), P^{\lambda}(\mathbb{H})$ or $P^{2}(\mathbb{O})$, then the first three non-vanishing Betti numbers $b_{i}$ of $\Omega(M)$ are $b_{0}, b_{a}$ and $b_{a+n-1}$. Here $a=1$ in the complex projective case, $a=3$ in the quaternionic projective case, $a=7$ in the Cayley projective case and $b_{a}=1$ in each case. If $M$ has the homology of $S^{n}$, then the first two non-vanishing Betti numbers of $\Omega(M)$ are $b_{0}$ and $b_{n-1}$.

From Theorem 2.11 and Proposition 2.15, we deduce the following statement: Let $M$ be a compact simply connected pointwise taut manifold, so that there is a point $p \in M$ for which each point of the first conjugate locus has the multiplicity one. Then the first three non-vanishing Betti numbers $b_{i}$ of the based loop space of $M$ are $b_{0}, b_{1}=1$ and $b_{n}$. According to Theorem 1.5, $M$ is Blaschke. Therefore, we can apply a result on Blaschke manifolds, which is due to Yang ([Ya]).

Theorem 2.16. Let $M^{2 \lambda}$ be a Blaschke manifold, so that the homology of $M$ is isomorphic to that of $P^{\lambda}(\mathbb{C})$. Then $M$ is homeomorphic to $P^{\lambda}(\mathbb{C})$. If $\lambda>2$, then $M$ is even diffeomorphic to $P^{\lambda}(\mathbb{C})$.

Hence, we obtain the following

Theorem 2.17. Let $M^{n}$ be a compact simply connected pointwise taut manifold, so that there is a point $p$ in $M$ for which each point of the first conjugate locus has the multiplicity one. Then $n$ is even (say, $n=2 \lambda)$ and $M$ is homeomorphic to $P^{\lambda}(\mathbb{C})$. If $n>4$, then $M$ is even diffeomorphic to $P^{\lambda}(\mathbb{C})$.

Next, we consider the spherical case $k=n-1$ in Theorem 2.1. Because $M$ is homeomorphic to $S^{n}$ according to that theorem, we deduce from Proposition 2.15 that the first non-vanishing Betti numbers of $\Omega(M)$ are $b_{0}$ and $b_{n-1}$. The first claim of Theorem 1.4 and our assumption about pointwise tautness imply together that $M$ is Blaschke. It is known that the spherical Blaschke conjecture has been solved by Green ([Gr]) in dimension two and by Berger and Kazdan in arbitrary dimension ([Be], p. 236):

Theorem 2.18. If $\left(S^{n}, g\right)$ is a Blaschke manifold, then $\left(S^{n}, g\right)$ is the standard sphere with constant sectional curvature. 
We conclude that $M$ is isometric to the standard sphere $\left(S^{n}, g\right)$ with constant sectional curvature.

Now we investigate the quaternionic case $k=3$. We know from Theorem 2.1 that the cohomology of $M^{n}$ is that of $P^{\lambda}(\mathbb{H})$, where $n=4 \lambda$. Proposition 2.15 implies that the first non-vanishing Betti numbers of $\Omega(M)$ are $b_{0}, b_{k}=1$ and $b_{k+n-1}$, as these are known to be the first three non-vanishing Betti numbers of $\Omega\left(P^{\lambda}(\mathbb{H})\right)$, see the paper of Terng and Thorbergsson ([TT]). Theorem 1.5 and pointwise tautness imply that $M$ is Blaschke. From a paper by Sato ([Sa]), we deduce that $M$ is homeomorphic to $P^{\lambda}(\mathbb{H})$.

Last but not least, we consider the Cayley case $k=7$. From Theorem 2.1, we know that the cohomology of $M^{n}$ is that of $P^{2}(\mathbb{O})$. The Cayley version of Proposition 2.15 implies that the first non-vanishing Betti numbers of $\Omega(M)$ are $b_{0}$, $b_{k}=1$ and $b_{k+n-1}$, as these are known to be the first three non-vanishing Betti numbers of $\Omega\left(P^{2}(\mathbb{O})\right)$. Theorem 1.5 and pointwise tautness again imply that $M$ is Blaschke. From a paper by Gluck and Warner ([GW]), we deduce that $M$ is homeomorphic to $P^{2}(\mathbb{O})$. Summarizing our results, we obtain the first theorem stated in the introduction.

\section{The three-dimensional case}

In this section we are going to apply the results of the previous section to the threedimensional case.

Let $M$ be a compact simply connected pointwise taut three-manifold. Obviously, the only possible multiplicities of conjugate points in $M$ are one and two. First, we assume that there is a point $p \in M$ so that each point in the first conjugate locus $C_{1}(p)$ has multiplicity one. Then the dimension of $M$ must be even due to the first theorem stated in the introduction, which contradicts our assumption $\operatorname{dim}(M)=3$.

Therefore, the only remaining possibility is the following: for each point $p \in M$, there is a point $q \in C_{1}(p)$ so that the multiplicity of $q$ is two. Theorem 3.3 implies now that $M$ is Blaschke and homeomorphic to $S^{3}$. Using Theorem 2.18, we immediately obtain the following

Theorem 3.1. Let $M$ be a compact simply connected pointwise taut three-manifold. Then $M$ is isometric to the standard sphere $S^{3}$.

Remark. Of course, the two-dimensional case can be trivially handled in the same way. One can immediately verify that a compact simply connected pointwise taut two-manifold is isometric to the standard sphere $S^{2}$. 


\section{The four-dimensional case}

In this section, we will prove some geometric and topological results about compact simply connected pointwise taut four-manifolds by means of studying the regular subset $C^{R}(p)$ of the first tangent conjugate locus $C_{1}(p)$, where $p$ is an arbitrary point. Theorem 2.2 implies that the conjugate points on a connected component of $C^{R}(p)$ have constant multiplicity and thus we can locally apply our techniques from Section 2 .

Let $M$ be a compact simply connected pointwise taut four-manifold. In this case, the possible multiplicities of conjugate points in $M$ are one, two and three. If we assume that for some point $p \in M$ there is a point $x \in C_{1}(p)$ which is of multiplicity three, then Theorem 1.4 implies that $M$ is homeomorphic to $S^{4}$.

Therefore, we can assume in the sequel that there are no points of multiplicity three in the first conjugate locus. If all the first conjugate points are of multiplicity one, then $M$ is homeomorphic to $P^{2}(\mathbb{C})$ due to Theorem 2.17. If all the points in the first conjugate locus were of multiplicity two, that would contradict the first theorem stated in the introduction. Therefore, we are left with the case where some of the first conjugate points are of multiplicity one and the others are of multiplicity two. An example of this situation is the manifold $S^{2} \times S^{2}$, endowed with the product metric.

As usual, let $C_{1}(p)$ denote the first conjugate locus, where $p$ is an arbitrary base point of a compact simply connected pointwise taut manifold $M$. Due to Theorem 2.10, $C_{1}(p)$ is homeomorphic to $S^{3}$. Let $x \in C_{1}(p)$ be a point of multiplicity two and $q=\exp _{p}(x)$. Then Theorem 1.2 implies that there is a compact two-dimensional critical submanifold $C r$ in the path space $\Omega_{p q}(M)$, its points being geodesics from $p$ to $q$ of common length $d$. We can map each of these geodesics $\left.\gamma\right|_{[0, d]} \in C r$ to the intersection point $d \gamma^{\prime}(0)$ of $C_{1}(p)$ with the ray $\exp _{p}^{-1}\left(\left.\gamma\right|_{[0, d]}\right)$. This map is a bijection, for $q$ is the first point conjugate to $p$ along any of these geodesics. The image of $C r$ under this map in $C_{1}(p)$ is a smooth two-dimensional manifold, as one can see by repeating the argument from the proof of Proposition 2.8. As a compact two-dimensional manifold embedded into the three-dimensional $C_{1}(p)$, the critical manifold $\mathrm{Cr}$ must be orientable. It is well known that every compact orientable two-dimensional manifold is a sphere or a connected sum of tori. Thus we have established the following

Lemma 4.1. Let $M$ be a compact simply connected pointwise taut four-manifold and $p$ be an arbitrary point in $M$. Let $x \in C_{1}(p)$ be a point of multiplicity two and $q=\exp _{p}(x)$. Then $x \in T(x) \subset C_{1}(p)$, where $T(x)$ is a sphere or a connected sum of tori and consists of conjugate points of multiplicity two.

Remark. Obviously, the statement above also holds for a compact simply connected four-manifold with a taut point $p$, as we did not use the tautness of other points. 
Now we are going to study how the set $C^{S}(p)$ of singular conjugate points partitions the first conjugate locus (recall that the latter is a three-dimensional sphere up to homeomorphy). Due to Lemma 2.4 , all points in $C^{S}(p)$ are of multiplicity two. Theorem 1.2 assures that $C^{S}(p)$ is a disjoint union of critical surfaces.

We are going to use the example $M=S^{2} \times S^{2}$ as our source of intuition. In this case, $C^{S}(p)$ consists of just one critical surface. This surface is a torus $T$ dividing the sphere $C_{1}(p)$ into two connected components, both of which are solid tori. We could view one of these as the "inner side" of $T$ and the other as the "outer side" of $T$, but in this case, they are homeomorphic to each other. In order to remedy this ambiguity and define the notion of the inner side, we choose an arbitrary multiplicity one conjugate point $\mathcal{I}$ in $C_{1}(p)$, where $\mathcal{I}$ stands for "infinity". Now we call the component of $C_{1}(p) \backslash T$, which does not contain $\mathcal{I}$, the inner side of $T$. The point $\mathcal{L}$ can not belong to $C^{S}(p)$ because each conjugate point of multiplicity one is regular, according to Lemma 2.4 .

In the general case, there can be more than two connected components in the set of regular conjugate points $C^{R}(p)=C_{1}(p) \backslash C^{S}(p)$. A priori, there could be even infinitely many (compact oriented) critical surfaces in $C^{S}(p)$. Any critical surface $T$ splits the topological sphere $C_{1}(p)$ into two connected components, and our choice of the infinity point indicates which of these two components to consider the inner side of $T$. If a subset of $C^{R}(p)$ is bounded by (only) one surface belonging to $C^{S}(p)$, then we call that subset an inner component of $C^{R}(p)$. Of course, not every critical surface in $C^{S}(p)$ gives rise to an inner component in $C^{R}(p)$. Though, one intuitively expects $C^{R}(p)$ to contain at least one inner component. Our next goal is to prove this fact. Before doing that, we use Theorem 1.2 to observe that the critical surfaces in $C^{S}(p)$ are always pairwise disjoint.

Lemma 4.2. Let $M$ be a compact simply connected four-manifold with a taut point $p \in M$. Let $C_{1}(p)$ be its first tangent conjugate locus and $C^{S}(p) \subset C_{1}(p)$ be the subset of singular conjugate points. Then there is an inner component in $C^{R}(p)$.

Proof. According to the above, $C^{S}(p)$ is a disjoint union of critical surfaces $T_{i}$, where $i \in I$. Apriori, the index set $I$ could be infinite. Let $T_{i}$ and $T_{j}$ be two of these surfaces. We write $T_{i} \ll T_{j}$, if $T_{i}$ lies in the inner side of $T_{j}$. It is straightforward to verify that $\ll$ is an equivalence relation. The set Crit of critical surfaces is partially ordered by this relation, but $\ll$ is not necessarily a total ordering on Crit. In fact, there can obviously be two tori in $C_{1}(p)$, neither of which is contained in the inner side of the other. Our goal is to prove that each totally ordered subset of Crit has a lower bound, so that we can apply Zorn's Lemma.

Let $\mathcal{O}$ be a totally ordered subset of Crit. We consider a monotonously decreasing sequence $\left(T_{k}\right)$ in $\mathcal{O}$ with the property that, for each $T \in \mathcal{O}$, there is a $T_{k}$ satisfying $T_{k} \ll T$. For each $k$, we choose an arbitrary point $x_{k} \in T_{k}$. Because $C_{1}(p)$ is 
compact, the sequence $\left(x_{k}\right)$ must possess a cluster point $x_{0}$. Theorem 2.6 implies that the set $C^{S}(p)$ of singular conjugate points is closed. Therefore, we have $x_{0} \in C^{S}(p)$ and the multiplicity of $x_{0}$ must be two. Let $T_{0}$ be the critical surface which contains $x_{0}$. By its construction, the point $x_{0}$ lies inside of each $T \in \mathcal{O}$. By continuity, $T_{0} \ll T$ for all $T \in \mathcal{O}$. We have shown that each totally ordered $\mathcal{O} \subset$ Crit has a lower bound. By Zorn's Lemma, Crit has a minimal element, which implies our claim.

Having established the existence of an inner component, we are going to deal with the question what is the multiplicity of the conjugate points in it. We recall that, due to Lemma 2.4, all singular conjugate points in $C_{1}(p)$ must be of multiplicity two. Now we show the converse statement for the inner components. (We remind the reader of our standard assumption for this section that there be no first conjugate points of multiplicity three).

Lemma 4.3. Let $M$ be a compact simply connected four-manifold with a taut point $p$. Let $A$ be an inner component of $C^{R}(p)$. Then all conjugate points in A have multiplicity one.

Proof. Due to Theorem 2.2, the conjugate points in any connected component of $C^{R}(p)$ have the same multiplicity. Assume that all points in $A$ are of multiplicity two. Let $\mathrm{Cut}_{A}=\exp _{p}(A)$ and $T_{A} \subset C^{S}(p)$ be the critical surface which bounds $A$. Then, due to Lemma 2.14, $\mathrm{Cut}_{A}$ is a one-dimensional manifold and the restriction $\exp _{p}: A \rightarrow \mathrm{Cut}_{A}$ is a fibration with fiber $S^{2}$. Moreover, the union of $\mathrm{Cut}_{A}$ and the point $\left\{\exp \left(T_{A}\right)\right\}$ is connected and compact. The well-known classification of onedimensional manifolds implies that $\mathrm{Cut}_{A}$ is an interval $I$. The fibration $\exp _{p}: A \rightarrow$ $\mathrm{Cut}_{A}$ is trivial, for $\mathrm{Cut}_{A}$ is simply connected. We deduce that $A=S^{2} \times I$. But on the other hand, $A$ is inner and therefore its boundary must be connected. This contradiction shows that all points in $A$ are of multiplicity one.

After our preparatory discussion of inner components, we are ready to make this concept work and obtain a purely geometric fact.

Proposition 4.4. Let $M$ be a compact simply connected four-manifold. Let $p \in M$ be a taut point. Then $p$ is conjugate to itself along a suitable geodesic. In particular, if $M$ is pointwise taut, then every point is conjugate to itself along a suitable geodesic.

Proof. Consider the first conjugate locus $C_{1}(p)$ in $T_{p}(M)$ and the subset $C^{S}(p)$ of singular conjugate points. According to Lemma 4.2, there is an inner component $A$ in $C_{1}(p) \backslash C^{S}(p)$, which is bounded by a critical surface $T_{A}$. Since the set $A \cap T_{A}$ is compact, the norm function attains a minimum and a maximum on this set. Theorem 1.2 implies that all the elements of the boundary $T_{A}$ have the same norm. Therefore, the norm function attains at least one of its extrema in $A$. Let $x_{0} \in A$ be such 
an extremal point and $S_{0}$ denote the critical circle passing through $x_{0}$. For each $x \in S_{0}$, the ray $r_{x}$ from the origin through $x$ must be orthogonal to $C_{1}(p)$ in $x$. From Theorem 1.2 we know that the points on $S_{0}$ have the same norm. Moreover, $\exp _{p}$ maps $S_{0}$ to one point in $M$, and we $\operatorname{set} q=\exp _{p}\left(S_{0}\right)$. Now we consider the restriction $\left.\exp _{p}\right|_{A}: A \rightarrow \operatorname{Cut}(p)$ of the exponential map. From Lemma 2.14 and Lemma 4.3, we deduce that the subset $\operatorname{Cut}_{A}=\exp _{p}(A) \subset \operatorname{Cut}(p)$ is a two-dimensional manifold. We write $T_{q}$ Cut for its tangent space at $q$. Using the Gauss Lemma, we realize that each of the geodesics $\exp _{p}(t x)$ for $x \in S_{0}$ is orthogonal to $T_{q}$ Cut at the point $q$. The unit direction vectors of these geodesics form a circle in $T_{q} M$, which thus lies in the two-dimensional orthogonal complement of $T_{q}$ Cut. We conclude that each of these geodesics comes back to $p$, because for each direction represented in this circle, the opposite direction also belongs to the circle. Because the point $p$ is taut and infinitely many geodesics emanating from $p$ return to $p$, that point must be conjugate to itself.

Remark. In the above theorem, we need not demand that there be no conjugate points of multiplicity three in the first conjugate locus, nor that there be a point of multiplicity two in the first conjugate locus, although these are our standard assumptions in the current section. The theorem can be proved in the same way if all the conjugate points in the first conjugate locus are of multiplicity one. If there is a conjugate point of multiplicity three in the first conjugate locus, then Theorem 1.4 and Theorem 2.18 show that $M$ is isometric to $S^{4}$, endowed with the canonical metric, proving our assertion about conjugate points.

Further results about pointwise taut four-manifolds can be achieved if one allows for an additional assumption. For example, one can show that a compact homogeneous pointwise taut four-manifold is locally symmetric by using the well-known classification of homogeneous four-manifolds and checking each of the homogeneous metrics for pointwise tautness in a rather straightforward case-by-case study.

\section{References}

[Be] A. Besse, Manifolds all of whose geodesics are closed. Ergeb. Math. Grenzgeb. 93, Springer-Verlag, New York 1987. Zbl 0387.53010 MR 0867684

[Bo1] R. Bott, Nondegenerate critical manifolds. Ann. of Math. 60 (1954), 248-261. Zbl 0058.09101 MR 0064399

[Bo2] R. Bott, An application of Morse theory to the topology of Lie groups. Bull. Soc. Math. France 84 (1956), 251-281. Zbl 0073.40001 MR 0087035

[BS] R. Bott and H. Samelson, Applications of the theory of Morse to symmetric spaces. Amer. J. Math. 80 (1958), 964-1029. Zbl 0101.39702 MR 0105694 
[BT] R. Bott and L. W. Tu, Differential forms in algebraic topology. Grad. Texts in Math. 82, Springer-Verlag, New-York, 1982. Zbl 0496.55001 MR 0658304

[CW] S. Carter and A. West, Tight and taut immersions. Proc. London Math. Soc. 25 (1972), 701-720. Zbl 0242.53029 MR 0314071

[dC] M.P. do Carmo, Riemannian geometry. Mathematics: Theory \& Applications, Birkhäuser Inc., Boston, MA, 1992. Zbl 0752.53001 MR 1138207

[GH] K. Grove and S. Halperin, Elliptic isometries, condition (C) and proper maps. Arch. Math. 56 (1991), 288-299. Zbl 0726.53030 MR 1091884

[Gr] L. W. Green, Auf Wiedersehensflächen. Ann. of Math. 78 (1963), 289-299. MR 0155271

[GW] H. Gluck and F. Warner, Division algebras, fibrations of spheres by great spheres and the topological determination of space by the gross behaviour of its geodesics. Duke Math. J. 50 (1983), 1041-1076. Zbl 0534.53039 MR 0726317

[Oz] T. Ozawa, On critical sets of distance functions of a taut submanifold. Math. Ann. 276 (1986), 91-96. Zbl 0583.53052 MR 0863709

[Pa] R. S. Palais, Morse theory on Hilbert manifolds. Topology 2 (1963), 299-340. Zbl 0122.10702 MR 0158410

[PS] R. S. Palais and S. Smale, A generalized Morse theory. Bull. Amer. Math. Soc. 70 (1964), 165-172. Zbl 0119.09201 MR 0158411

[PT] R. S. Palais, and C. L. Terng, Critical point theory and submanifold geometry. Lecture Notes in Math. 1353, Springer-Verlag, Berlin, New York 1988. Zbl 0658.49001 MR 0972503

[Sa] H. Sato, On topological Blaschke conjecture, I. In Geometry of Geodesics and related topics (Tokio 1982), Adv. Stud. Pure Math. 3, North-Holland, Amsterdam 1984, 231-238. Zbl 0557.53023 MR 0758656

[Se] J.-P. Serre, Homologie singulière des espaces fibrés. Ann. of Math. 54 (1951), 425-505. Zbl 0045.26003 MR 0045386

[TT] C. L. Terng and G. Thorbergsson, Taut immersions into complete Riemannian manifolds. In Tight and taut submanifolds (Berkeley, CA, 1994), Math. Sci. Res. Inst. Publ. 32, Cambridge University Press, Cambridge 1997, 181-228. Zbl 0906.53043 MR 1486873

[Wa1] F. W. Warner, The conjugate locus of a Riemannian manifold. Amer. J. Math. 87 (1965), 575-604. Zbl 0129.36002 MR 0208534

[Wa2] F. W. Warner, Conjugate loci of constant order. Ann. of Math. 86 (1967), 192-212. Zbl 0172.23003 MR 0214005

[Wo] J. A. Wolf, Differentiable fibre spaces and mappings compatible with Riemannian metrics. Michigan Math. J. 11 (1964), 65-70. Zbl 0116.39202 MR 0159285

[Ya] C. T. Yang, Smooth great circle fibration and an application to the topological Blaschke conjecture. Trans. Amer. Math. Soc. 320 (1990), 507-524. Zbl 0704.57018 MR 1028766

Received April 14, 2003

Michael Leitschkis, Mathematisches Institut der Universität zu Köln, Weyertal 86-90, 50931

Köln, Germany

E-mail: mleitchk@mi.uni-koeln.de 